\title{
Letter To The Editor:
}

I enjoyed reading the article by Dr. Heller and team concerning Transscutaneous Pacing Devices. The study was well done and very informative. There are a few comments and questions I have concerning the paper and other issues involving emergency TCP.

In my review of the current literature concerning TCP, I have found a well-done but rarely cited study entitled" Transcutaneous pacing: Experience with the Zoll noninvasive temporary pacemaker.' This study found that 33 of 35 patients were able to be paced by the Zoll TCP. Of these 35 patients, 24 pacing attempts were done in the acute setting with 22 successful captures and 21 survived to discharge.

This study used patients between 15 and 90 years of age with a median being 68 . For this group, the median milliamps required for capture was 55. Of the conscious patients, 16 of 29 complained of chest pain, 9 requiring analgesia and/or sedation. Most complained of pain at 60-80 milliamps. These results tend to confirm Rottmans suggestion concerning the Zoll unit consistency. ${ }^{2}$ A question posed by Gunderson deserves some thought concerning first-line use of TCP before atropine. Consider the case of a symptomatic, bradycardic patient in the presence of acute MI, especially in the prehospital setting. Many of us have seen these patients given atropine, then a bradycardia of 40 becomes a tachycardia of 120 . Along with the rate change is noted an increase in chest pain, ST segment elevation, and patient apprehension. In this setting, would it not be wiser to TCP the patient at $60-70 \mathrm{BPM}$, transport to the emergency department, and then make a decision concerning atropine? Just food for thought. The question of need vs. limited use is quite appropriate. In larger, multitiered systems, placing these units strategically within the system may be a solution. Of course, this is not always possible. Cummins, et al., also describes a solution by suggesting multipurpose units. The newly released LifePak 10 may be an answer. The question of TCP in the prehospital and emergency setting still leaves great room for research and opinion. Perhaps the industry got off on the wrong foot by originally marketing the units as a godsend for cardiac arrest vs. the condition it really seems to benefit: symptomatic bradycardia with palpable pulse.

Harold C. Cohen, EMT-P

Lieutenant, Baltimore County Fire Department

\section{References}

1. Madsen JK, Meibom J, Videbak R, et al: Transcutaneous pacing: Experience with the Zoll noninvasive temporary pacemaker. Am Heart J, 1988; 116:7.

2. Rottman S, Editorial Comments on Heller MB, et al. Prehosp Disaster Med. 1989; 4:19-20.

3. Cummins RO, Graves JR, Larsen MP, et al: Prehospital transcutaneous pacing of significant bradycardia by paramedics: Clinical and system effectiveness. Prehosp Disaster Med. 1989; 4:70 (abst). 